\title{
Study of indium tin oxide--MoS 2 interface by atom probe tomography
}

\author{
Manuel Ramos, Departamento de Física y Matemáticas, Instituto de Ingeniería y Tecnología, Universidad Autónoma de Cd. Juárez, Avenida del Charro 450 \\ N, Cd. Juárez, Chihuahua, C.P. 32310, Mexico; Institute for Applied Materials (IAM-WK), Karlsruhe Institute of Technology (KIT), Engelbert-Arnold-Str. 4, \\ D-76131 Karlsruhe, Germany \\ John Nogan, Center for Integrated Nanotechnologies, 1101 Eubank BIdg. SE, Albuquerque, NM 87110, USA \\ Torben Boll, Institute for Applied Materials (IAM-WK), Karlsruhe Institute of Technology (KIT), Engelbert-Arnold-Str. 4, D-76131 Karlsruhe, Germany; \\ Karlsruhe Nano Micro Facility (KNMF), Karlsruhe Institute of Technology (KIT), Hermann-von-Helmholtz-Platz 1, D-76344 Eggenstein-Leopoldshafen, \\ Germany
}

Sandra Kauffmann-Weiss, Institute for Applied Materials (IAM-WK), Karlsruhe Institute of Technology (KIT), Engelbert-Arnold-Str. 4, D-76131 Karlsruhe, Germany

Claudia A. Rodriguez-Gonzalez and Jose L. Enriquez-Carrejo, Departamento de Física y Matemáticas, Instituto de Ingeniería y Tecnología, Universidad Autónoma de Cd. Juárez, Avenida del Charro 450 N, Cd. Juárez, Chihuahua, C.P. 32310, Mexico Martin Heilmaier, Institute for Applied Materials (IAM-WK), Karlsruhe Institute of Technology (KIT), Engelbert-Arnold-Str. 4, D-76131 Karlsruhe, Germany

Address all correspondence to Manuel Ramos at manuel.ramos@uacj.mx

(Received 8 July 2019; accepted 30 October 2019)

\begin{abstract}
The molybdenum disulfide $\left(\mathrm{MoS}_{2}\right)$ and indium tin oxide (ITO) interface were studied by atom probe tomography (APT). Raman spectroscopy, scanning electron microscopy, and grazing-incidence $\mathrm{x}$-ray diffraction measurements were performed as complementary characterization. Results confirm that nanowires plated shape with the $\langle 110\rangle$-orientation are aligned perpendicular to the ITO film with principal reflections at (002), (100), (101), (201), and Raman spectroscopy vibrational modes at $E_{2 \mathrm{~g}}^{1}$ at $378 \mathrm{~cm}^{-1}$ and $A_{1 \mathrm{~g}}$ at $407 \mathrm{~cm}^{-1}$ correspond to $2 \mathrm{H}-\mathrm{MoS}_{2}$. APT reveals $\mathrm{MoS}^{+2}, \mathrm{MoS}^{+3}$ as predominant evaporated molecular ions on the sample, indicating no significant diffusion/segregation of Mo or S species within the ITO layer.
\end{abstract}

\section{Introduction}

Layered semiconductor materials have attracted considerable attention in the past 10 years for the fabrication of electronic devices such as transistors, energy storage batteries, and other photovoltaic and catalytic engineering applications. ${ }^{[1,2]}$ The $\mathrm{MoS}_{2}$ crystal structure was resolved by Pauling and Dickinson, ${ }^{[3,4]}$ who were able to determine S-Mo-S sandwich layers as stacked in three layers over the $c$-axis by a van der Waals weak bonding creating a unit cell. ${ }^{[4]} \mathrm{MoS}_{2}$ lattice parameters are always $a=b=3.17 \AA$, whereas the $c$-axis varies according to the found polyforms $2 \mathrm{H}-\mathrm{MoS}_{2}$ (hexagonal, $c=12.3 \AA$ ), $3 \mathrm{R}-\mathrm{MoS}_{2}$ (rhombohedral, $c=18.3 \AA$ ), and $1 \mathrm{~T}-\mathrm{MoS}_{2}$ (trigonal). ${ }^{[5,6]} \mathrm{MoS}_{2}$ theoretically predicted and experimentally verified band gaps range from 0.8 to $1.9 \mathrm{eV}$ as extensively discussed in the literature. ${ }^{[7]}$ Observations of the $\mathrm{MoS}_{2}$ structure on the transmission electron microscopy (TEM) level were first made by Chianelli et al. confirming an average $\sim 0.62 \mathrm{~nm}$ distance between $\mathrm{MoS}_{2}$ layers. ${ }^{[8]}$ Garcia et al. performed a TEM experiment to determine radiation damage under Cs-corrected TEM observations encountering that $80 \mathrm{kV}$ is appropriate to achieve a good observation on single layers, which were able not to obtain any three-dimensional (3D) information. ${ }^{[9]}$ And, using the computer software, $\mathrm{Wu}$ et al. estimated the sample thickness versus contrast by ADF-STEM and SAED simulations. ${ }^{[10]}$ Even though TEM is a highly sophisticated microanalysis tool, it is only possible to achieve 2D imaging, ${ }^{[1]}$ and while electron tomography by TEM can be applied to obtain volume distribution of $\mathrm{MoS}_{2}$ thin films and particles, it is limited to $-30^{\circ}$ to $+30^{\circ}$ of probe tilting, as described by Kong et al. ${ }^{[12]}$ Compared to that, laserassisted atom probe tomography (APT) is a characterization technique to determine the chemical and spatial resolution at the 3D nanometric scale. For example, APT was used to determine titanium homogeneous spatial (3D) distribution within a $\mathrm{MoS}_{2}$ matrix on magnetron-sputtered thin films, as reported by Singh et al. ${ }^{[13]}$ Also, it was used to obtain a spatial distribution on $\mathrm{SiGe} / \mathrm{Si} / \mathrm{SiGe}$ interfaces, in order to determine any migration and volume distribution between species. ${ }^{[14]}$ Here, APT measurements of molybdenum disulfide $\left(\mathrm{MoS}_{2}\right)$ layers on indium tin oxide (ITO) are presented. ITO was selected for the electrical contact layer because of its low resistivity $n$-type semiconductor behavior having $2-4 \times 10^{-4} \Omega \mathrm{cm}$ together with other beneficial electronic properties. The optimum electrical properties of ITO can be achieved through post-deposition annealing of the film at temperatures between 300 and $500^{\circ} \mathrm{C}$, and it is highly used as a transparent contact material for the integration and fabrication of electronic devices like graphic display 


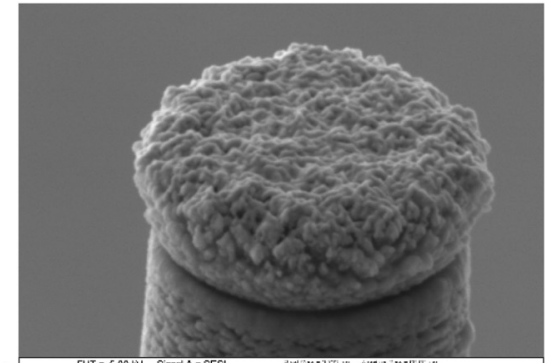

(a)

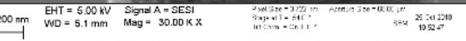

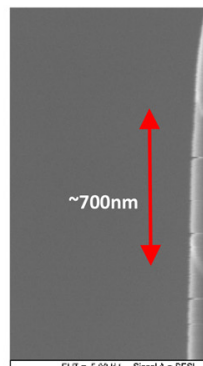

(b)

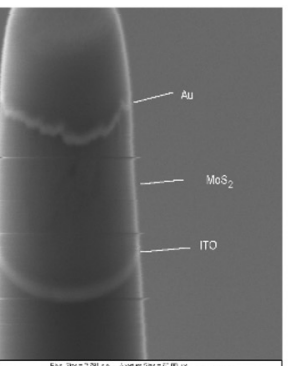

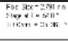

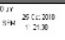

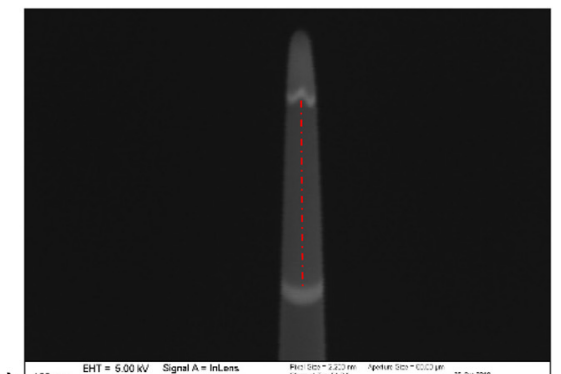

(c)

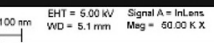

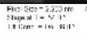

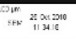

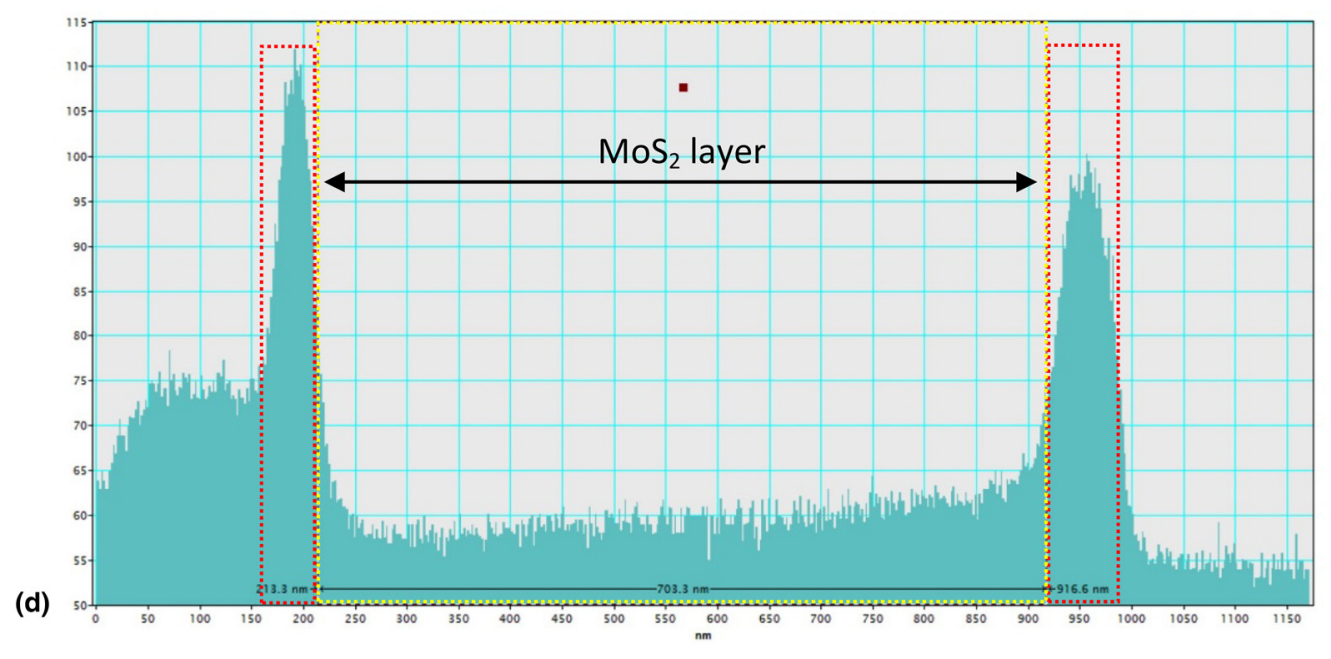

Figure 1. (a) SEM image for ITO-MoS 2 as-deposited over commercial APT pristine coupons. (b) SEM image of the tiny needle as completed by FIB is possible to determine the layers; by contrast, the red arrow indicates some porosity. (c) A sharper needle used to perform the APT measurements. (d) Brightness profile along the tip axis between left-ITO center-MoS 2 and right-ITO coating [as denoted by a red dotted line on (c)]. Each portion of ITO measures $\sim 100 \mathrm{~nm}$, while the $\mathrm{MoS}_{2}$ layer measures $\sim 700 \mathrm{~nm}$.

panels, photovoltaic panels, and nanotransistors. ${ }^{[15-19]}$ In our study, a metal-semiconducting interface was studied using APT to determine any possible migration/precipitation of indium (In), tin ( $\mathrm{Sn})$, oxygen $\left(\mathrm{O}_{2}\right)$, molybdenum $(\mathrm{Mo})$, and sulfur $(\mathrm{S})$ species, which in principle could affect electron mobility. The long-term motivation of this work is the fundamental understanding of layered $\mathrm{MoS}_{2}$ templates for low-cost solar cells fabrication.

\section{Materials and methods}

ITO and $\mathrm{MoS}_{2}$ were deposited directly onto commercial APT coupons $\left(\mathrm{Cameca}^{\odot}\right)$ as substrates using a Kurt J. Lesker PVD-75 RF-magnetron sputtering located in a class 1000 clean room (S1). The ITO deposition period was $1800 \mathrm{~s}$ with a sputter gas mixture of $90 \% \mathrm{Ar}$ and $10 \% \mathrm{O}_{2}$ at $20^{\circ} \mathrm{C}, 3 \mathrm{mT}$ at $145 \mathrm{~W}$ DC. To maintain consistently low ITO resistivity, $\mathrm{O}_{2}$ was only introduced during the deposition period of the processing sequence. Prior to and following the deposition phase, $100 \%$ Ar was utilized to stabilize the target's surface prior to deposition and in preparation for subsequent runs. After deposition, the ITO film was subjected to a rapid thermal annealing process, $300^{\circ} \mathrm{C}$ for $20 \mathrm{~min}$ at atmospheric pressure using a
$100 \mathrm{sccm}$ gas mixture of $3.8 \% \mathrm{H}_{2} /$ Balance $\mathrm{N}_{2}$. The subsequent $\mathrm{MoS}_{2}$ deposition period was $3600 \mathrm{~s}$ using $100 \% \mathrm{Ar}$ at $20^{\circ} \mathrm{C}$, $3 \mathrm{mT}$ at an applied power of $275 \mathrm{~W}$ DC. The aforementioned deposition conditions achieved an $\mathrm{MoS}_{2}$ layer with a thickness of approximately $700 \mathrm{~nm}$. The film surface was observed using a scanning electron microscopy (SEM) model Hitachi SU5500 equipped with energy-dispersive $\mathrm{x}$-ray unit and operated at $20 \mathrm{kV}$ with $8 \mathrm{~A}$ to avoid sample damage. The 3D spatial distribution of $\mathrm{S}$, Mo, In, Sn, and $\mathrm{O}_{2}$ was studied by an APT technique, using a Cameca $^{\circledR}$ LEAP $4000 \mathrm{X}$ high-resolution system, equipped with a UV laser $(\lambda \sim 355 \mathrm{~nm})$. All measurements were taken at a set temperature of $50 \mathrm{~K}$ with an evaporation rate of 0.2 and a laser frequency of $100 \mathrm{kHz}$. The laser beam was set to $20 \mathrm{pJ} / \mathrm{V}$, and all data were reconstructed from SEM images using the Cameca IVAS $^{\circledR}$ 3.6.14 package. Additional samples were prepared by directly coating the microtip coupons provided by the CAMECA with the multilayer sputtering system. The samples were prepared by annular milling with the aid of the focused ion-beam (FIB) instrument models FEI ${ }^{\odot}$ Strata or Zeiss Auriga, both equipped with dual beam. The surface was protected with an additional platinum layer deposited from a precursor gas within the SEM-FIBs to 

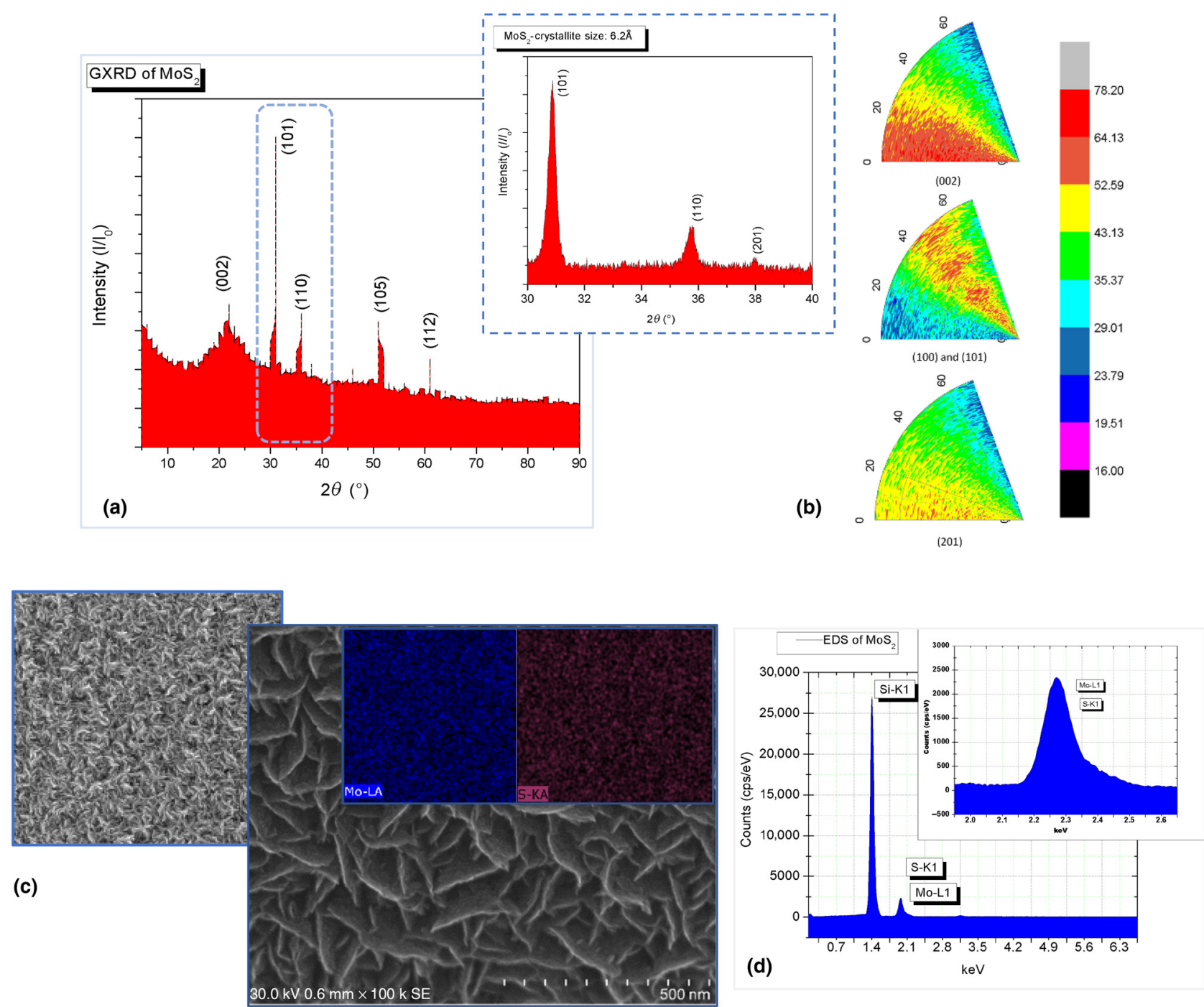

Figure 2. (a) GIXRD pattern and pole figures for $\mathrm{MoS}_{2}$ as-deposited over ITO; (b) pole figures and predominant diffraction for (101) indicating a vertical alignment of $\mathrm{MoS}_{2}$ crystallites in agreement with the literature ${ }^{[22]}$; (c) SEM image for the $\mathrm{MoS}_{2}$ surface; it is possible to observe vertical laminar structures typical of $\mathrm{MoS}_{2}$ (inset: colored signals of the Mo and S content as obtained by EDS), in agreement with Lince and Fleischauer ${ }^{[22]}$; and (d) energy-dispersive $\mathrm{x}$-ray spectroscopy (EDS) taken from $\mathrm{MoS}_{2}$ surfaces during the SEM survey (inset: an enlarge region near $2.1 \mathrm{keV}$ where S-KA1 and Mo-LA1 signals overlap).

reduce amorphization of the $\mathrm{MoS}_{2}$ film matrix due to Ga-ion beam radiation; FIB was carried out with 1000-100 pA ion beam gun power. The grazing-incidence $\mathrm{x}$-ray diffraction (GIXRD) was performed in a Rigaku Smart Lab with a HyPix 3000 detector and $\mathrm{Cu} \mathrm{K}_{\alpha}$ radiation source. The sample was aligned using the $\mathrm{Rx}-\mathrm{Ry}$ stage, parallel beam with PA-optics, and an incident angle of $0.2^{\circ}$ or $0.5^{\circ}$. The detector was in the point detector 0D mode, texture analysis was completed with $5^{\circ}$ Soller slits on beam side and detector side, slits on detector side are open $(10 \mathrm{~mm})$, speed $50 \% \mathrm{~min}$, the detector in the area 2D mode.

\section{Results and discussion}

Using SEM during FIB needle preparation for APT measurements, it was possible to observe a typical high porosity
$\mathrm{MoS}_{2}$ film matrix surface (Fig. 1(a)). A direct deposit on pristine APT coupons was done due to the fragility seen during the FIB lift-out process from deposits made on $\mathrm{SiO}_{2}$ (images are presented in the Supplementary material); thus, a reduction of amorphization over ITO- $\mathrm{MoS}_{2}$ interface was achieved. Amorphization is typical, mainly in semiconductors as extensively described by Bogdanowicz et al. for silicon (Si) and gallium nitride $(\mathrm{GaN})$ thin films during the FIB lift-out process. ${ }^{[20]}$ Vertical nucleation and growth oriented along the $\langle 110\rangle-\mathrm{MoS}_{2}$ direction of neddle-like crystallites over the silicon substrate using similar RF-sputtering conditions have been previously reported by Ramos et al., ${ }^{[21]}$ and it is presented in Figs. 1(a)1(d). The GIXRD principal reflections at (002) which corresponds to $2 \mathrm{H}-\mathrm{MoS}_{2}$ stacks (wire thickness), (100) and (101) and (201) indicate space group 2H-R3 $\mathrm{m}$ and average crystallite 


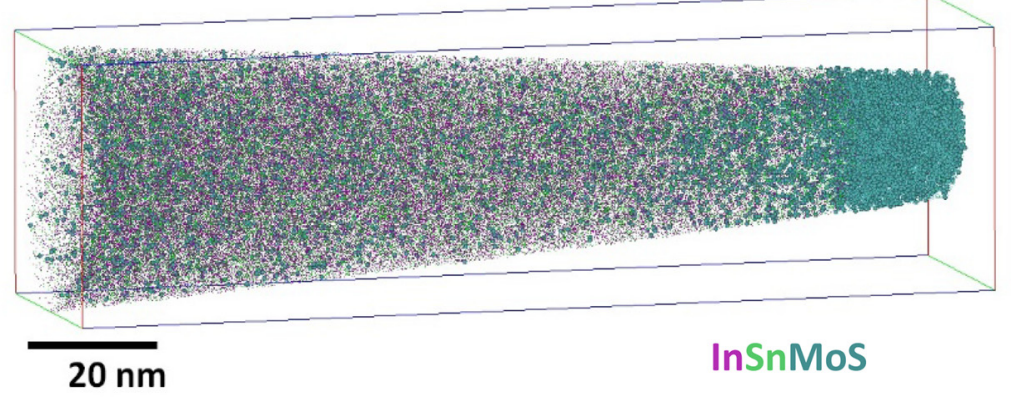

(a)

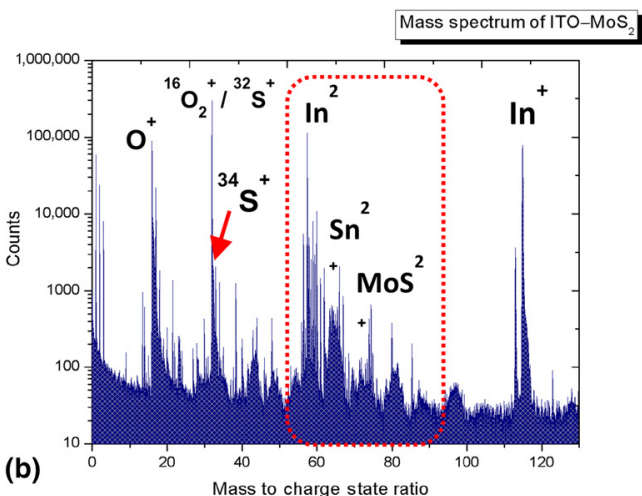

Figure 3. (a) APT distribution for the ITO-MoS interface. $\mathrm{MoS}_{2}$ is displayed as small spheres ${ }^{16} \mathrm{~S}$ cannot be distinguished from ${ }^{16} \mathrm{O}_{2}$ easily in the mass spectrum, as they both have a signal at 32 amu. Every single dot represents one atom, and for visibility, only $20 \%$ of In atoms are displayed with no enrichment found within the ITO-MoS 2 interface. (b) Mass spectrum of the ITO-MoS $\mathrm{S}_{2}$ interface. Due to the complexity of the mass spectra, only the peaks near 60 amu were identified as MoS contributions mainly and used for the APT reconstruction. Other peaks were identified as $0, \mathrm{Sn}, \ln , \mathrm{SnO}, \operatorname{InO}, \mathrm{SnS}, \operatorname{InS}, \mathrm{SO}$, and Mo.

size of $6.216 \AA$ and $d_{(101)}=2.838 \AA$, as shown in Fig. 2(a) (insets: enlargement at (101)-principal reflection). This is in agreement with the results of Lince and Fleischauer and Jiang et al. for heterogenous $\mathrm{MoS}_{2} / \mathrm{WS}_{2}$ heterojunctions. ${ }^{[22,23]}$ And we found no substantial evidence of $1 \mathrm{~T}$ and $2 \mathrm{H}$ mixedphase formed during sputtering, as recently reported by Sarma et al. ${ }^{[24]}$ SEM images indicate that rugosity [Fig. 2(c)] and elemental analysis by surface energy-dispersive $\mathrm{x}$-ray emission confirmed the presence of $\mathrm{S}$ and Mo as shown in Fig. 2(d). A full description of the APT technique is beyond this report, but in brief it consists of placing a sample to undergo an ionizing evaporation process by the laser pulse at a high electric field under UHV conditions and low temperatures. Then, the potential energy of an ion leaving the sample's surface is due to the shape of the field almost entirely converted into kinetic energy of $1 / 2 m v^{2}$ in the close vicinity of the tip. Thus, to obtain the mass-spectra signal, the relationship given by $m / n=2 e / d^{2}\left(\mathrm{~V}_{\mathrm{dc}}\right) t^{2}$ is used to analyze the mass- to charge-state ratio $(\mathrm{m} / \mathrm{n})$ of ions evaporated; $n$ is the number of electrons removed from an ion, $e$ the electron charge $\left(-1.62 \times 10^{-19} \mathrm{C}\right), V_{\mathrm{dc}}$ is the applied voltage, $m$ is the atomic mass, and the speed of an ion is given as $v=d / t$, with $d$ being the distance detector-tip and $t$ is the time of flight (a schematic of test set-up is presented in Supplementary material). ${ }^{[25]}$ The results from APT measurements indicate a well-defined interface between $\mathrm{MoS}_{2}$ and ITO layers as shown in Fig. 3(a); allowing to observe a well-defined chemical contact of $\mathrm{MoS}_{2}$ and the ITO matrix (Supplementary video), with little, to no intermixing within chemical species. From the time-of-flight mass spectra, as shown in Fig. 3(b), it was possible to determine that sulfur and molybdenum tend to evaporate as MoS molecular ions $\left(\mathrm{MoS}^{+2}, \mathrm{MoS}^{+3}, \mathrm{MoS}_{3}{ }^{+2}\right.$, and $\left.\mathrm{MoS}^{+1}\right)$. Due to its typical peak fingerprint, these molecules, as well as $\mathrm{Sn}$, can be identified easily and are used to track the respective element distributions. Indium has only one major isotope, but it does not show any overlaps with any other possible peaks from
ITO and $\mathrm{MoS}_{2}$, and thus the corresponding atoms can be identified with certainty. The most problematic overlap for this material is between $\mathrm{S}^{+}$and $\mathrm{O}_{2}{ }^{+}$. Here, it is necessary to disregard the main peak for both 32 amu and the minor peak at 33 amu. By contrast, the peak at 34 amu almost exclusively stems from $\mathrm{S}$ and can be used to track its position. The same problem exists for the double-charged ion $\mathrm{S}^{2+}$. However, the isotope at 17 amu cannot be used because it overlaps with $\mathrm{OH}^{+}$. In Fig. 4, the proxigram profile through the 50 at.\% indium isosurface is shown, and it was utilized to identify the ITO- $\mathrm{MoS}_{2}$ interface concentration along the vertical growth direction and more detailed volume reconstruction is presented in Fig. 4(b). It was calculated with a bin size of $0.1 \mathrm{~nm}$ as a function of the distance from the isosurface. The high spread of the $\mathrm{S}$ and $\mathrm{O}$ concentration results from the necessity to disregard the main $\mathrm{S} / \mathrm{O}_{2}$ peak at $32 \mathrm{amu}$ in the mass spectrum. Together with the subsequent background correction, this causes the originally-in absolute values - small errors for $\mathrm{S}$ and $\mathrm{O}$ concentrations to spread to values that can even be negative for $\mathrm{O}$. The continuous lines through the data points illustrate the concentration gradient without this effect.

In our APT study, it was mainly intended to be able to distinguish the existence of $1 \mathrm{~T}$ and $2 \mathrm{H}$ phases within the $\mathrm{MoS}_{2}$ film matrix with a spatial distribution map-based approach as described by Boll et al. ${ }^{[26,27]}$ and Geiser et al., ${ }^{[28]}$ since evidence of its existence was reported by x-ray photon spectroscopy (XPS). ${ }^{[2]}$ However, the aberrations during ions trajectory, due to limited APT precision in the laser mode, made it difficult to achieve an accurate reconstruction of the tip surface. ${ }^{[29]}$ Moreover, the high fragility of the tips, specially at high vacuum conditions, did not allow us to achieve a large collection of time-of-flight events to complete statistical analysis. The spatial distribution of S and Mo is given in Fig. 4(c), which indicates no segregation or migration of In, $\mathrm{Sn}$, or $\mathrm{O}_{2}$ from the ITO layer. And, using Raman surface spectroscopy, we were able to confirm the formation of the $2 \mathrm{H}-\mathrm{MoS}_{2}$ 


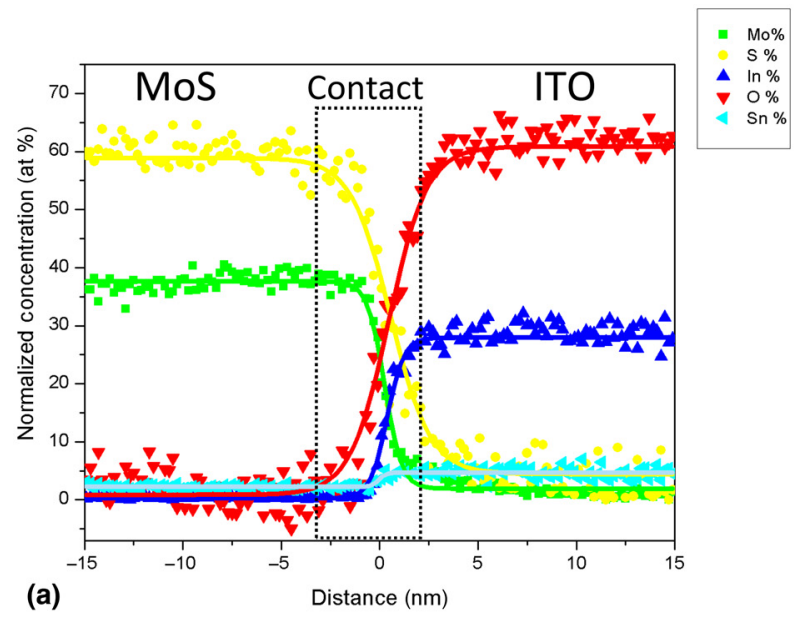

(a)

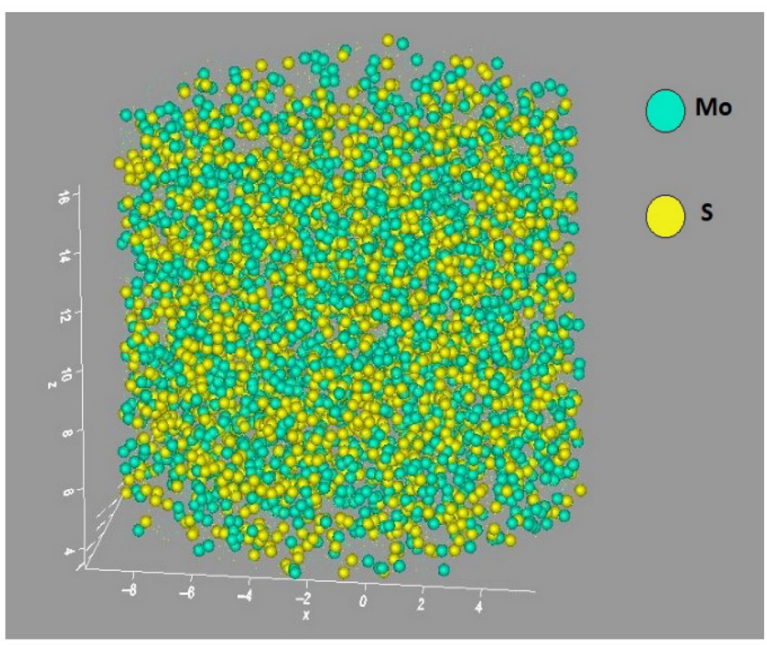

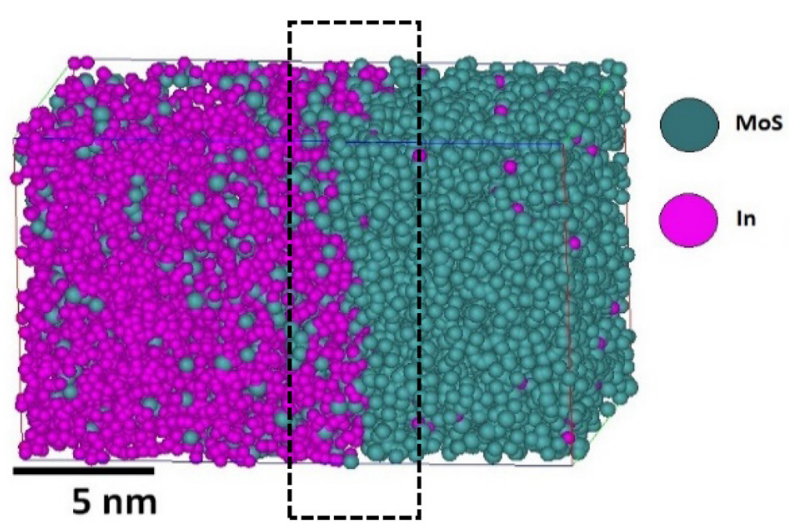

(b)

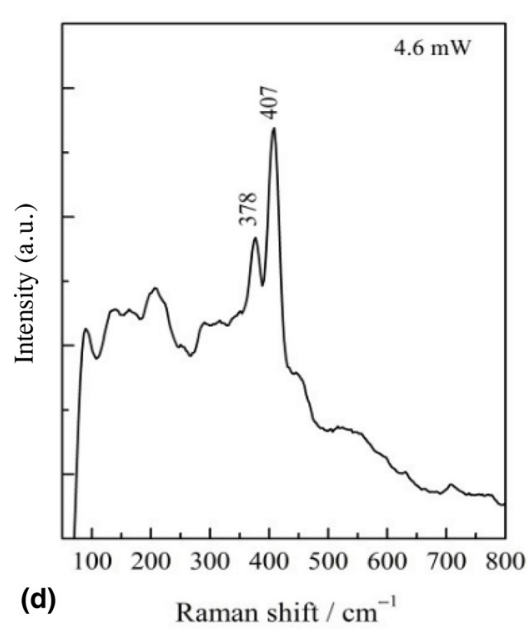

Figure 4. (a) Proxigram of relative composition for $\mathrm{S}, \mathrm{O}_{2}$, Mo, In, and $\mathrm{Sn}$ as data processed taken from the interaction at the interface. (b) APT time-of-flight volume reconstruction for ITO and $\mathrm{MoS}_{2}$ interface. (c) Spatial atomic reconstruction for the $\mathrm{MoS}_{2}$ matrix from APT measurements. No specific features were encountered. (d) Raman spectroscopy performed over the $M_{0} S_{2}$ film surface, typical two main vibrational modes at $E_{2 g}^{1}$ at $378 \mathrm{~cm}^{-1}$ and $A_{1 \mathrm{~g}}$ at $407 \mathrm{~cm}^{-1}$ are found in agreement with the literature. ${ }^{[12,24]}$

phase, due to modes of vibration at $\mathrm{E}_{2 \mathrm{~g}}^{1}$ at $378 \mathrm{~cm}^{-1}$ and $\mathrm{A}_{1 \mathrm{~g}}$ at $407 \mathrm{~cm}^{-1}$ at the surface as presented in Fig. 4(d), and previously discussed in Ref. [21].

\section{Conclusions}

We successfully presented RF-sputtering of $2 \mathrm{H}-\mathrm{MoS}_{2}$ thin films over the ITO substrate from commercial $99.9 \%$ targets. Our results from Raman spectroscopy and GIXRD indicated large colonies of $2 \mathrm{H}-\mathrm{MoS}_{2}$ crystallites with epitaxial growth along $\langle 101\rangle$. By SEM, it was possible to observe large colonies of flakes with porosity within. APT allowed us to determine spatial distribution reconstruction of the ITO- $\mathrm{MoS}_{2}$ interfaces, and this reconstruction shows no indication of segregation of any specific element within the film matrix and interface. The proxigram concentration profiles confirmed the uniform composition of $\mathrm{MoS}_{2}$ and ITO layers. The obtained information from APT measurements indicates that ITO can serve as an ideal transparent contact material, suggesting that the semiconducting electronic structure properties will be retained in multilayers of $\mathrm{MoS}_{2}-$ ITO.

\section{Supplementary material}

The supplementary material for this article can be found at https://doi.org/10.1557/mrc.2019.150.

\section{Acknowledgments}

The principal author, M. Ramos, thanks the Karlsruhe Micro and Nano Facility (KNMF) of Karlsruhe Institute of Technology for the usage of atom probe tomography and FIB instruments under proposal 2018-020-022609. The KNMF grants measurement time to external users for proposals pertinent to KNMF goals. The authors would like to express their 
gratitude to Delphine Chassaing from KIT for the APT-FIB preparation. Universidad Autónoma de Ciudad Juárez for financial support by PIVA-2017 project titled: "Fabricación de plantillas metálico-orgánicas: Una ruta hacia celdas solares más eficientes." And to UACJ-PFCE-1337 scholar travel award from UACJ. Part of this work was performed at the Center for Integrated Nanotechnologies, an Office of Science User Facility operated for the U.S. Department of Energy (DOE) Office of Science. Sandia National Laboratories is a multi-program laboratory managed and operated by Sandia Corporation, a fully owned subsidiary of Lockheed Martin Corporation, for the U.S. Department of Energy's National Nuclear Security Administration under contract DE-AC0494AL85000.

\section{References}

1. A. Kumar Singha, P. Kumar, D.J. Late, A. Kumar, S. Patel, and J. Singh: 2D layered transition metal dichalcogenides $\left(\mathrm{MoS}_{2}\right)$ : synthesis, applications and theoretical aspects. Appl. Mater. Today 13, 242-270 (2018).

2. X. Li and H. Zhu: Two-dimensional $\mathrm{MoS}_{2}$ : properties, preparation, and applications. J. Materiomics 1, 33-44 (2015).

3. The Nobel Prize in Chemistry 1954. NobelPrize.org. Nobel Media AB 2018. Tue. 11 Dec 2018.

4. R.G. Dickinson and L. Pauling: The crystal structure of molybdenite. J. Am. Chem. Soc. 45, 1466-1471 (1923).

5. M.A. Ramos, R. Chianelli, J.L. Enríquez-Carrejo, G.A. Gonzalez, and G. Berhault: Metallic states by angular dependence in $2 \mathrm{H}-\mathrm{MoS}_{2}$ slabs. Comp. Mater. Sci. 84, 18-22 (2014).

6. M. Ramos, F. Galindo-Hernández, I. Arslan, T. Sanders, and J.M. Domínguez: Electron tomography and fractal aspects of $\mathrm{MoS}_{2}$ and $\mathrm{MoS}_{2} / \mathrm{Co}$ spheres. Sci. Rep. 7, 12322.

7. N. Perea-López, Z. Lin, N.R. Pradhan, A. Iñiguez-Rábago, A.L. Elías, A. McCreary, J. Lou, P.M. Ajayan, H. Terrones, L. Balicas, and M. Terrones: CVD-grown monolayered $\mathrm{MoS}_{2}$ as an effective photosensor operating at low-voltage. 2D Mater. 1, 011004 (2014).

8. R.R. Chianelli, A.F. Ruppert, M. José-Yacamán, and A. Váquez-Zavala: HREM studies of layered transition metal sulfide catalytic materials. Catal. Today 23, 269-281 (1995).

9. A. Garcia, A.M. Raya, M.M. Mariscal, R. Esparza, M. Herrera, S.I. Molina, G. Scavello, P.L. Galindo, M. José-Yacamán, and A. Ponce: Analysis of electron beam damage of exfoliated $\mathrm{MoS}_{2}$ sheets and quantitative HAADF-STEM imaging. Ultramicroscopy 146, 33-38 (2014).

10. R.J. Wu, M.L. Odlyzko, and K. Andre Mkhoyan: Determining the thickness of atomically thin $\mathrm{MoS}_{2}$ and $\mathrm{WS}_{2}$ in the TEM. Ultramicroscopy 147, 8-20 (2014).

11. R.F. Egerton, R. McLeod, F. Wang, and M. Malac: Basic questions related to electron-induced sputtering in the TEM. Ultramicroscopy 110, 991997 (2010).

12.D. Kong, H. Wang, J.J. Cha, M. Pasta, K.J. Koski, J. Yao, and Y. Cui: Synthesis of $\mathrm{MoS}_{2}$ and $\mathrm{MoSe}_{2}$ films with vertically aligned layers. Nano Lett. 13, 1341-1347 (2013).

13. H. Singh, K.C. Mutyala, R.D. Evans, and G.L. Doll: An atom probe tomography investigation of Ti-MoS 2 and $\mathrm{MoS}_{2}-\mathrm{Sb}_{2} \mathrm{O}_{3}$-Au films. J. Mater. Res. 32, 1710-1717 (2017).

14. O. Dyck, D.N. Leonard, L.F. Edge, C.A. Jackson, E.J. Pritchett, P.W. Deelman, and J.D. Poplawsky: Accurate quantification of Si/SiGe interface profiles via atom probe tomography. Adv. Mater. Interfaces 4, 1700622 (2017).

15. J. Szász, S. Seils, D. Klotz, H. Störmer, M. Heilmaier, D. Gerthsen, H. Yokokawa, and E. Ivers-Tiffée: High-resolution studies on nanoscaled $\mathrm{Ni} / Y S Z$ anodes. Chem. Mater. 29, 5113-5123 (2017).

16. H. Kim and C.M. Gilmore: Electrical, optical, and structural properties of indium-tin-oxide thin films for organic light-emitting devices. J. Appl. Phys. 86, 6451-6461 (1999).
17. U. Betz, M. Kharrazi Olsson, J. Marthy, M.F. Escolá, and F. Atamny: Thin films engineering of indium tin oxide: large area flat panel displays application. Surf. Coat. Technol. 200, 5751-5759 (2006).

18. S. Qiao, B. Zhang, K. Feng, R. Cong, W. Yu, G. Fu, and S. Wang: Large lateral photovoltage observed in $\mathrm{MoS}_{2}$ thickness-modulated ITO/MoS $/$ p-Si heterojunctions. ACS Appl. Mater. Interf. 9, 18377-18387 (2017).

19. M. Marikkannan, M. Subramanian, J. Mayand, M. Tanemura, V. Vishnukanthan, and J.M. Pearce: Effect of ambient combinations of argon, oxygen, and hydrogen on the properties of DC magnetron sputtered indium tin oxide films. AIP Adv. 5, 017128 (2015).

20. J. Bogdanowicz, A. Kumara, C. Fleischmann, M. Gilbert, J. Houard, A. Vella, and W. Vandervorst: Laser-assisted atom probe tomography of semiconductors: the impact of the focused-ion beam specimen preparation. Ultramicroscopy 188, 19-23 (2018).

21.M. Ramos, J. Nogan, M. Ortíz-Díaz, J.L. Enriquez-Carrejo, C.A. Rodríguez-González, J. Mireles-Jr-Garcia, C. Ornelas, and A. Hurtado-Macias: Mechanical properties of RF-sputtering $\mathrm{MoS}_{2}$ thin films. Surf. Topogr.: Metrol. Prop. 5, 025003 (2017).

22. J.R. Lince and P.D. Fleischauer: Crystallinity of RF-sputtered $\mathrm{MoS}_{2}$ films. J. Mater. Res. 2, 827-838 (1987).

23. X. Jiang, B. Sun, Y. Song, M. Dou, J. Ji, and F. Wang: One-pot synthesis of $\mathrm{MoS}_{2} / \mathrm{WS}_{2}$ ultrathin nanoflakes with vertically aligned structure on indium tin oxide as a photocathode for enhanced photo-assistant electrochemical hydrogen evolution reaction. RSC Adv. 7, 49309 (2017).

24. S. Sarma and S. Chandra Ray: Trigonal (1T) and hexagonal $(2 \mathrm{H})$ mixed phases $\mathrm{MoS}_{2}$ thin films. App. Surf. Sci. 474, 227-231 (2019).

25. T.F. Kelly and M.K. Miller: Atom probe tomography. Rev. Sci. Inst. 78, 031101 (2007). doi: 10.1063/1.2709758.

26. T. Boll, T. Al-Kassab, Y. Yuan, and Z.-G. Liu: Investigation of the site occupation of atoms in pure and doped $\mathrm{TiA} 1 / \mathrm{Ti}_{3} \mathrm{Al}$ intermetallic. Ultramicroscopy 107, 796-801 (2007).

27. T. Boll, Z.-Y. Zhu, T. Al-Kassab, and U. Schwingenschlög: Atom probe tomography simulations and density functional theory calculations of bonding energies in $\mathrm{Cu}_{3} \mathrm{Au}$. Microsc. Microanal. 18, 964-970 (2012).

28. B.P. Geiser, T.F. Kelly, D.J. Larson, J. Schneir, and J.P. Roberts: Spatial distribution maps for atom probe tomography. Microsc. Microanal. 13, 437-447 (2007).

29. B. Gault, M.P. Moody, F. de Geuser, G. Tsafnat, A. La Fontaine, L.T. Stephenson, D. Haley, and S.P. Ringer: Advances in the calibration of atom probe tomographic reconstruction. J. Appl. Phys. 105, 34913 (2009). 